\title{
Identification of dry and fresh cocoon silk
}

\begin{abstract}
Cocoon silks, one of most important textile materials, have different classification, such as fresh cocoon silk and dry cocoon silk. Different cocoon silks quality is different, also affects the subsequent processing. In order to distinguish fresh and dry cocoon silks, many tests are done such as cohesive property, cleanliness, and structural analysis etc. The results show CV values are 0.161728 and 0.131093 for fresh cocoon silk and dry cocoon silk, respectively. The fluctuation level of CV value for fresh cocoon silk is larger than dry cocoon silk. Cohesive properties of fresh cocoon silk and dry cocoon silk are 64 and 102. Meanwhile, cleanliness and clean inspection values of dry cocoon silk are 93.85 and 98.6 higher than fresh cocoon silk. Furthermore, the secondary structures of these two cocoon silks are similar. In addition, the breaking strength and extension at break of fresh cocoon silk is lower than dry cocoon silk, for $6.183 \pm 1.371 \mathrm{cN} / \mathrm{dtex}$ and $27.54 \pm 8.53 \%$. Therefore, for same grade cocoon silks, it can make comprehensively evaluation between appearance (including defects and cleanliness etc.) and structural aspects.
\end{abstract}

Keywords: cocoon silk, cohesive property, structural
Volume 2 Issue 6 - 2017

\author{
infaJ Ming, ', Qingtian Pan, ${ }^{2,3}$ Fukui Pan,' Xin \\ Ning,' Baoqi Zuo 2,3 \\ 'Industrial Research Institute of Nonwovens and Technical \\ Textiles, Qingdao University, China \\ ${ }^{2}$ National Engineering Laboratory for Modern Silk, Soochow \\ University, China \\ ${ }^{3}$ College of Textile and Clothing Engineering, Soochow \\ University, China
}

Correspondence: Jinfa Ming, Industrial Research Institute of Nonwovens and Technical Textiles, College of Textiles \& Clothing, Qingdao University, Qingdao, 26607I, China, Tel 8653285953358, Fax 8653285953076,

Email mingjinfa@qdu.edu.cn,jinfa.ming@gmail.com

Received: August 28, 2017| Published: September II, 2017

\section{Introduction}

Silkworm cocoon silk fiber is composed of two parts: fibroin and sericin, accounting for about 75 and $25 \mathrm{wt} \%$, respectively. Fibroin contains highly ordered crystalline domains and lessordered intermediate domains that result in silk's unique mechanical properties such as strength and flexibility. ${ }^{1}$ Sericin is amorphous acting as adhesive binder to maintain the fine filaments of fibroin and the overall structural integrity of cocoon. ${ }^{2}$ Silk fibers are one of most important materials extensively used in textile industry. Silk processing from cocoons to the finished products consists of a series of steps. Degumming in reeling is a key process during which sericin is removed by thermo-chemical treatment of cocoon. Since degumming imposes a relatively harsh environment on silk, the possibility of changes occurring in silk microstructure and mechanical properties etc. must be considered.

At present, many degumming methods in reeling are used to remove sericin and obtain raw silk. In reeling mill, the current silk reeling uses a circulating cocoon cooking machine that allows water to penetrate into cocoons at high temperature and operate on the principle of vacuum infiltration. All the cocoon cooking and reeling processes are automated. This process is called dry cocoon silk reeling, and its obtained silk is dry cocoon silk. However, fresh cocoon silk reeling is direct vacuum permeation at low temperature, ellipsis of cooking and drying process. Compared with dry cocoon silk reeling, fresh cocoon silk reeling has many advantages such as fast processing speed, high yield and low energy consumption etc. As early as 1984, Hisashi attempted to reel silk directly from frozen cocoons in order to avoid the complex process of cocoon drying and cooking. ${ }^{3}$ Thus, in these reeling processes, sericin swelling and water penetration into cocoon shell affect cocoon reeling and the quality of the raw silk. In this paper, to deeply understand the differences of fresh and dry cocoon silks, cohesive property, cleanliness, and structural characterization etc. are characterized.

\section{Experimental section}

\section{Materials}

Dry and fresh cocoon silk were bought from Shandong province,
China. The specification of silk was 20/22 dtex. All tests were performed under constant temperature and humidity condition $\left(20^{\circ} \mathrm{C}\right.$ and $65 \% \mathrm{RH})$.

\section{Defect extraction and evenness of cocoon silks}

Defect morphology and silk evenness are detected by autoinspection machine. ${ }^{4}$ After threshold segmentation and morphology processing operation, raw silk has been extracted from images, and then defects and evenness of raw silk are tested. During image acquisition, raw silk is moving at speed of $10 \mathrm{mmin}^{-1}$, and each sample length is $2000 \mathrm{~m}$.

\section{Cohesive property and cleanliness}

The cohesive property of cocoon silk is tested according to $\mathrm{GB} /$ T1798-2008 standard. At the same time, the cleanliness of cocoon silk is tested according to GB/T1797-2008 standard. In these tests, an average of twenty measurements was reported.

\section{Structural characterization}

The secondary structure of samples was analyzed by FTIR on Nicolet5700 (Thermo Nicolet Company, USA) in absorbance mode. For each measurement, each spectrum was obtained by the performance of 32 scans with the wave number ranging from 400 to $4000 \mathrm{~cm}^{-1}$ with a resolution of $4 \mathrm{~cm}^{-1}$. Moreover, in order to investigate the crystalline structure of samples, X-ray diffraction experiment was measured on X Pert-Pro MPD (PANalytical, Netherlands) in transmittance mode. The incident beam wavelength was $0.154 \mathrm{~nm}$. The intensity was finally corrected for changes in the incident beam intensity, sample absorption, and background.

\section{Mechanical property}

The mechanical properties of different cocoon silk were measured by automatic tensile tester (model 3365 electronic strength tester, Instron, Boston, USA). Before test, samples were kept for $24 \mathrm{~h}$ at standard atmospheric conditions $\left(20^{\circ} \mathrm{C}\right.$ and $\left.65 \% \mathrm{RH}\right)$. During test process, distance between grips and test speeds were set to $250 \mathrm{~mm}$ and $10 \mathrm{~mm} \mathrm{~min}^{-1}$, respectively. At the same time, the pre-tension was $0.2 \mathrm{cN}$. An average of thirty measurements was reported as the mean \pm standard deviation for each sample. 


\section{Results and discussion}

\section{Defects and evenness evaluation of fibers}

Generally, as any product in the market claims its quality, cocoon silks also have their own quality. For silk industry, on downstream processing enterprises and consumers' perspective, cocoon silk producer can produce high quality silk. Nowadays, due to the difference reeling methods, there are two kinds of cocoon silks in the market, namely: fresh cocoon silk and dry cocoon silk. The quality of silk products such as strength, dying etc. is directly influenced by these two different cocoon silks. In order to distinguish these two cocoon silks from their appearance, seriplane test system and electronic tester can be used. ${ }^{5}$ Traditionally, raw cocoon silk is inspected manually for defects or so-called seriplane inspection. This method, greatly influenced by human factors, is strong subjective, poor reproducibility and accuracy of experimental results. Hence, silk electronic detection system is used in our experiment. This electronic detection system mainly includes image acquisition system (charge coupled device line scan camera, light resource, and telecentric lens etc.), computer image processing and result output. ${ }^{4}$

Figure 1 depicts the typical defect fragments obtained using silk electronic detection system. As can be seen, many loops and hairness are appeared on the surface of cocoon silk, especially fresh cocoon silk. The number of defects is 478 per $2000 \mathrm{~m}$. However, dry fresh cocoon silk has less, only for 4 per $2000 \mathrm{~m}$. This is mainly related to silk reeling. Furthermore, coefficient of variation (CV \%) is adopted to characterize the evenness of silk fibers in this electronic detection method. The calculation formula of $\mathrm{CV}$ is as follows:

$$
\mathrm{CV}(\%)=\frac{1}{\bar{x}} \sqrt{\frac{1}{n} \sum_{i=1}^{n}\left(x_{i}-\bar{x}\right)^{2}} \times 100 \%
$$

$\bar{x}:$ The average value of diameters

$\mathrm{n}$ : The total number of diameters

$x_{i}$ :The $i$-th value of diameter

Note: CV is a relative value and no unit. In this paper, we use the number of occupied pixels obtained by Halcon to calculate silk diameter rather than other method.

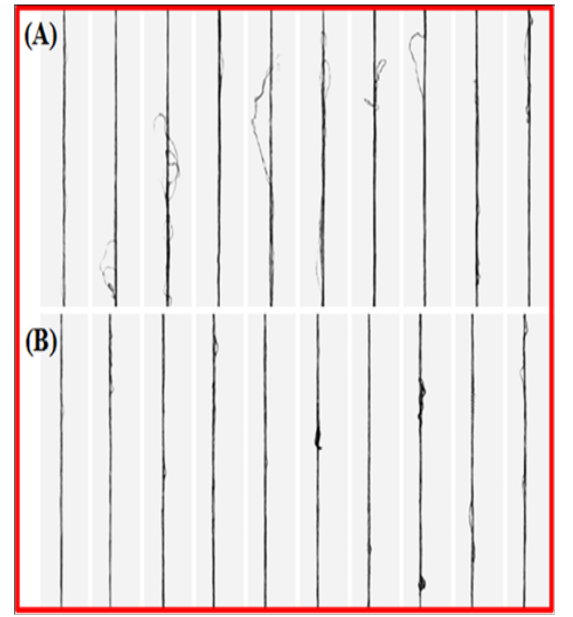

Figure I Typical defect fragments obtained by silk electronic detection system: (A) fresh cocoon silk; (B) dry cocoon silk.
Through calculation, CV values are 0.161728 and 0.131093 for fresh cocoon silk and dry cocoon silk, respectively. Fresh and dry cocoon silks are obtained through different reeling process. For fresh cocoon silk, silk reeling is direct vacuum permeation at low temperature, ellipsis of cooking and drying process. Sericin is less removed and its structure is loose. However, dry cocoon silk is obtained through cooking in boiling water and drying under high temperature. The degumming degree of dry cocoon silk is more serious than fresh cocoon silk. Therefore, the fluctuation level of CV value for fresh cocoon silk is larger than dry cocoon silk.

\section{Cohesive property and cleanliness of cocoon silk}

The cohesive property of cocoon silk is tested according to the standard GB/T1798-2008 raw silk experiment method. Table 1 showed the cohesive properties of fresh cocoon silk and dry cocoon silk are 64 and 102, respectively. The reasons for the significant difference are as follows: Firstly, silkworm cocoon silk is composed of silk fibroin protein coated by sericin proteins to glue fibroin brins together in forming the cocoon. ${ }^{6}$ Sericins are adhesive proteins that account for $25-30 \%$ of the total silkworm cocoon by weight. ${ }^{7}$ Due to sericin adhesion, cocoon silk has good cohesive property. Next, dry cocoon silk is reeling from cocoons treated by boiling and high temperature drying. At drying process, the dry temperature and time is $90-110^{\circ} \mathrm{C}$ and $5-6.5 \mathrm{~h}$. Sericin is denatured, its crystallinity is increased and the cohesive property is enhanced. However, fresh cocoon silk is obtained from non-cooking cocoons treated by vacuum permeation. In reeling process, the temperature is about $40-80^{\circ} \mathrm{C}$. Sericin has a smaller degeneration, and its structure is loose. Hence, the cohesive property of fresh cocoon silk is lower than dry cocoon silk.

Additionally, the cleanliness of cocoon silk is also tested according to the standard GB/T1797-2008 raw silk. Cleanliness and clean inspection values of fresh cocoon silk are 60 and 71.45. Meanwhile, cleanliness and clean inspection values of dry cocoon silk are 93.85 and 98.6. The results indicate the defect of dry cocoon silk is lower than fresh cocoon silk, namely dry cocoon silk has better quality, which is consistent with Figure 1 result.

\section{Structural characterization of cocoon silk}

Structural characterization is also a means to distinguish cocoon silk materials. Figure 2 depicts XRD and FTIR results of cocoon silk. Dry cocoon silk exhibited $\beta$-sheet structure, have $9.7^{\circ}, 20.3^{\circ}$, and $24.1^{\circ}$, respectively (Figure $2 \mathrm{a}$ ). ${ }^{8}$ At the same time, Figure $2 \mathrm{~b}$ shows the diffraction peaks at $9.9^{\circ}, 20.3^{\circ}$, and $24.1^{\circ}$, attributing to $\beta$-sheet structure. The results indicate the secondary structure of fresh and dry cocoon silk is similar (Table 2).

Structure changes in different cocoon silks are characterized by FTIR (Figure 2b). IR spectral region within $1700-1500 \mathrm{~cm}^{-1}$ was assigned to the peptide backbone of amide I $\left(1700-1600 \mathrm{~cm}^{-1}\right)$ and amide II (1600-1500 $\left.\mathrm{cm}^{-1}\right)$ absorptions; the amide III region was from $1350-1200 \mathrm{~cm}^{-1} .^{9-11}$ These infrared spectral regions have been used to analysis different secondary structure of silk. In this work, all samples are shown the main peaks at 1701,1630 and $1511 \mathrm{~cm}^{-}$ ${ }^{1}$, attributing to $\beta$-sheet structure. However, the relative ratios of different secondary conformations were calculated from the amide I region by deconvulation of IR spectra. The contents of random coil, $\alpha$-helix, $\beta$-turn, and $\beta$-sheet are $31.71 \%, 20.02 \%, 25.40 \%$, and $22.87 \%$, respectively, for dry cocoon silk. Moreover, the contents of random coil, $\alpha$-helix, $\beta$-turn, and $\beta$-sheet are $30.34 \%, 20.84 \%$, 
$24.64 \%$, and $24.18 \%$, for fresh cocoon silk. Thus it can be seen that the main components of different cocoon silks are also similar. Subtle structural differences cannot be used to distinguish fresh and dry cocoon silks.

\section{Mechanical property of cocoon silk}

The results of mechanical tests are shown in Table 3. The breaking

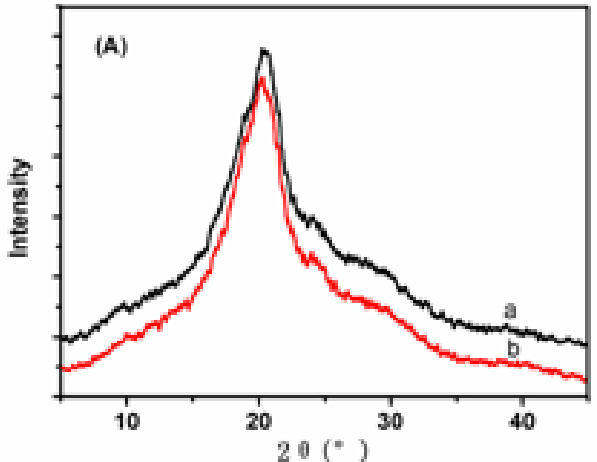

strength and extension at break are $6.315 \pm 1.104 \mathrm{cN} / \mathrm{dtex}$ and $29.67 \pm 7.54 \%$, for dry cocoon silk. However, the breaking strength and extension at break of fresh cocoon silk is lower than dry cocoon silk, for $6.183 \pm 1.371 \mathrm{cN} / \mathrm{dtex}$ and $27.54 \pm 8.53 \%$. The different in mechanical properties is related to cocoon silk diameter uniformity and its crystalline structure.

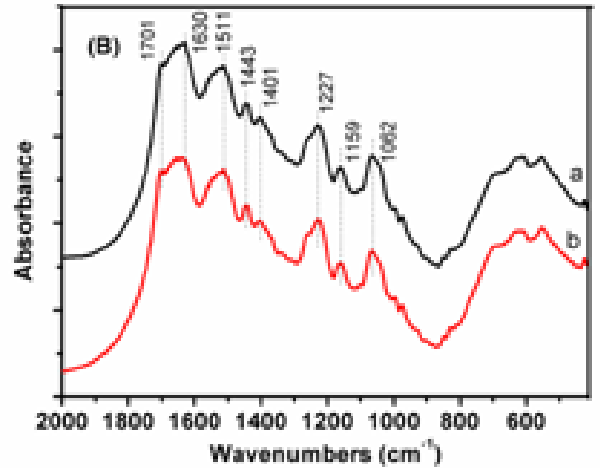

Figure 2 (A) XRD and (B) FTIR of different cocoon silks: (a) dry cocoon silk; (b) fresh cocoon silk.

Table I Cohesive property of fresh and dry cocoon silks

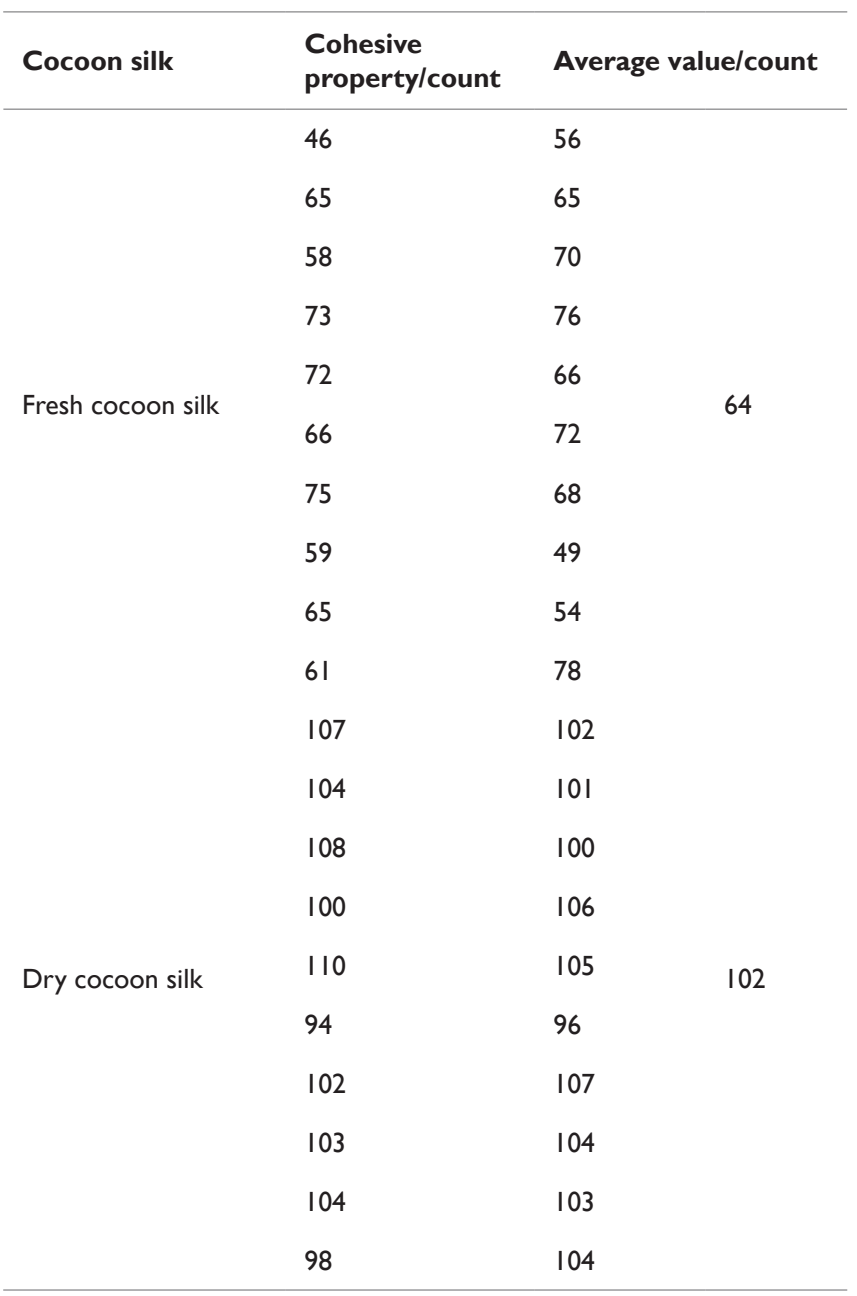

Table $\mathbf{2}$ The relative ratio of secondary structure in different cocoon silks

\begin{tabular}{lll}
\hline Assignment & $\begin{array}{l}\text { Dry } \\
\text { cocoon silk } \\
(\%)\end{array}$ & $\begin{array}{l}\text { Fresh } \\
\text { cocoon silk } \\
(\%)\end{array}$ \\
\hline $\begin{array}{l}\text { Silk II, } \beta \text {-sheet }\left(1610-1635 \mathrm{~cm}^{-1}, 1695-\right. \\
\left.\text { I700 } \mathrm{cm}^{-1}\right)\end{array}$ & 22.87 & 24.18 \\
Random coil $\left(1635-1645 \mathrm{~cm}^{-1}\right)$ & $31.7 \mathrm{I}$ & 30.34 \\
$\begin{array}{l}\text { Silk I, type II } \beta \text {-turn }\left(1647-1654 \mathrm{~cm}^{-1}\right) \\
\begin{array}{l}\alpha-h e l i x, \text { turns and bends }(1658-1695 \\
\left.\mathrm{cm}^{-1}\right)\end{array}\end{array}$ & 25.4 & 24.64 \\
\hline
\end{tabular}

Table 3 Mechanical properties of dry and fresh cocoon silk

\begin{tabular}{lll}
\hline Cocoon silk & $\begin{array}{l}\text { Breaking strength } \\
(\mathbf{c n} / \text { dtex })\end{array}$ & $\begin{array}{l}\text { Extension at } \\
\text { break (\%) }\end{array}$ \\
\hline Dry cocoon silk & $6.315 \pm 1.104$ & $29.67 \pm 7.54$ \\
Fresh cocoon silk & $6.183 \pm 1.37 \mathrm{I}$ & $27.54 \pm 8.53$ \\
\hline
\end{tabular}

\section{Conclusion}

Cocoon silks are one of most important materials extensively used in textile industry. Silk processing from cocoon to products consists of a series of steps, especially silk reeling. Different silk reeling methods are produced different silks, such as fresh cocoon silk and dry cocoon silk. In order to distinguish these two cocoon silks, cohesive property, cleanliness, and structural characterization etc are characterized. The results show that $\mathrm{CV}$ values are 0.161728 and 0.131093 for fresh cocoon silk and dry cocoon silk, respectively. The fluctuation level of $\mathrm{CV}$ value for fresh cocoon silk is larger than dry cocoon silk. Cohesive properties of fresh cocoon silk and dry cocoon silk are 64 and 102. Meanwhile, cleanliness and clean inspection values of dry cocoon silk are 93.85 and 98.6 higher than fresh cocoon silk. XRD and FFTIR results exhibit the secondary structure of these two cocoon silks are similar. Additionally, the breaking strength and extension at break of 
fresh cocoon silk is lower than dry cocoon silk, for $6.183 \pm 1.371 \mathrm{cN} /$ dtex and $27.54 \pm 8.53 \%$. Therefore, for the same grade of fresh and dry cocoon silks, people can make a distinction between appearance (including defects and cleanliness etc.) and structural aspects.

\section{Acknowledgements}

The authors thank Zheng Yang (Shandong Fiber Inspection Bureau, China) for the provision of cohesive property and cleanliness test and Qingtian Pan (Soochow University, China) for the provision of defect image extraction and analysis.

This work was financially supported by National Natural Science Foundation of Shandong Province (BS2015CL017), China Postdoctoral Science Foundation (2016M592141), Shandong Postdoctoral Innovation Project (201603067), Qingdao Innovation Project (17-1-1-68-jch), the Open Project Program of Key Laboratory of Eco-textiles, Ministry of Education, Jiangnan University (No. KLET1511), the Cooperation Project of Zhongyuan University of Technology (20163702011482), and Industrial Research Institute of Nonwovens \& Technical Textiles, Qingdao University.

\section{Conflict of interest}

a. This manuscript is original work, and has not been copyrighted or published previously.

b. No other financial support or incentive has been provided for this manuscript. (All financial support is listed in this paper.)

c. I am one author signing on behalf of all co-authors of this manuscript.

\section{References}

1. Horan RL, Antle $\mathrm{K}$, Collette AL, et al. In vitro degradation of silk fibroin. Biomaterials. 2005;26(17):3385-3393.

2. Jiang P, Liu HF, Wang $\mathrm{CH}$, et al. Tensile behavior and morphology of differently degummed silkworm cocoon silk fibers. Mater Lett. 2006;60(7):919-925.

3. Cao TT, Wang YJ, Zhang YQ. Silk reeling silkworm cocoon in strongly alkaline electrolyzed water as a sericin swelling agent at low temperature. J Text I. 2014;105(5):502-508.

4. Wang C, Li JJ, Chen M, et al. The obtainment and recognition of raw silk defects based on machine vision and image analysis. $J$ Text $I$. 2016;107(3):316-326.

5. Xu JM, Wu DP, Zhou Y, et al. Raw silk quality index comparison between electronic tester and seriplane test system. J Fiber Bioeng Inform. 2009;7(3):339-348.

6. Jin HJ, Kaplan DL. Mechanism of silk processing in insects and spiders. Nature. 2003;424(6952):1057-1061.

7. Rockwood DN, Preda RC, Yücel T, et al. Materials fabrication from Bombyx mori silk fibroin. Nat Protoc. 2011;6(10):1612-1631.

8. Ming JF, Pan FK, Zuo BQ. Influence factors analysis on the formation of silk I structure. Int J Biol Macromol. 2015;75:398-401.

9. Hu X, Kaplan D, Cebe P. Dynamic protein water relationships during beta sheet formation. Macromolecules. 2008;41(11):3939-3948.

10. Lu Q, Hu X, Wang X, et al. Water insoluble silk films with silk I structure. Acta Biomater. 2010;6(4):1380-1387.

11. Hu X, Kaplan D, Cebe P. Determining beta-sheet crystallinity in fibrous proteins by thermal analysis and infrared spectroscopy. Macromolecules.2006;39(18):6161-6170. 